\title{
ARTIFICIAL INTELLIGENCE: A FUTURE EXTRA LIMB OF OUR HEALTH CARE MARKETING FACILITIES
}

\section{HARIS RAFIQI \& SANA FAROOQ}

B. Engineering (Deakin University Australia) Hons, MBA (University of Nottingham), United Kingdom

M.D.S Pediatric and Preventive Dentistry; Tutor, Government Dental College and Hospital, Srinagar, Kashmir

\begin{abstract}
Nowadays Artificial intelligence has become an emerging marketing health campaign that helps in the diagnosis of health problems, the treatment recommendation to be followed, performing administrative activities many times outperforming healthcare tasks better than humans on a large section of the population. It provides a revolution to the current marketing strategies as well as creates entirely new ways of delivering valuable services to the customers. The tool of artificial intelligence has supported this global pandemic and the medical and the scientific community has high hopes that this data science will help in confronting the coronavirus thus proving as a beneficial marketing strategy.

KEYWORDS: Artificial Intelligence \& Coronavirus
\end{abstract}

Received: Jun 25, 2021; Accepted: Jul 15, 2021; Published: Aug 07, 2021; Paper Id: IJESRDEC20214

\section{INTRODUCTION}

Artificial intelligence (AI) is a branch of data science that leads fiction to reality by aiming at building smart machines that can mimic human behaviour and perform human actions but it lags the cognition of the human mind as it performs instruction fed activities. Gabriel (2019) stated that AI being a digital platform enables creativity, innovation and the ability to use digital technology to solve the years old traditional problems. ${ }^{1}$

In 2000 Robotic Surgery in the field of gynecology, prostate and head and neck surgery was approved in USA which gave surgeons superpowers by improving their ability to visualise, create more precision and use minimally invasive incisions though the important decisions are still made by surgeons. ${ }^{2}$

In healthcare, Artificial intelligence not only helps in tracking the crisis of COVID pandemic at the medical level but also at the molecular as well as epidemiological level thus helping in facilitating the research on the virus for the development of proper treatment regimes, preventive strategies, prevent future outbreak and drug and vaccine development thus reducing the workload off the shoulders of healthcare workers. ${ }^{3}$ A base triage system help in reducing the burden from medical practitioners and health care staff by automating several processes like providing training to practitioners, finalising the mode of treatment and care by analysing clinical data, digitalization of the patients' reports, minimising the contact with the patients distant monitoring of patients who didn't need hospital visits like asymptomatic patients or with mild symptoms.

Though AI systems will not replace the human resource of clinicians on a large scale but they will rather augment efforts taken for the care of patients. The healthcare jobs most likely to be benefited deal with digital information, radiology, pathology rather than those where direct contact with the patient is a must. ${ }^{4}$ 


\section{PRESENT AND THE FUTURE}

Nowadays $98 \%$ of marketers think of executing AI completely but literature research done in 2017 only $20 \%$ of the marketers have implemented one or more types of AI solutions. ${ }^{5}$ The major applications include neural networks, reasoning, an expert system and in practically upgrading the already outdated methods of marketing. $n$

Presently two types of AI can be categorised that is weak AI and strong AI where weak AI is able to emulate the human logic by analysing a large amount of data in contrast to strong AI which think machines to be conscience driven having an ability to think. ${ }^{6}$

AI brought a 360 degree revolution as it helped in developing sales and marketing strategies which include pricing, promotion, distribution, product planning, development and more importantly its use in digital platform, advertisement function and customer relationship management.

In the heath care industry, electronic health care record developers (EHR) play an instrumental role by using artificial intelligence to create more interfaces and automate some routine procedures that consume so much time of the user like clinical documentation (by voice recognition and dictation), order entry, sorting, helping in prioritising the task that truly require the clinicians' attention thus turning EHR into a reliable risk predictor ${ }^{7} \mathrm{AI}$ helps in containing the risk of antibiotic resistance because EHR can help in identifying the infection patterns, highlighting the patients at risk even before they show the symptoms and thus creating faster and accurate alerts for health care providers.

In the present COVID pandemic application of more advanced forms of automatic detection and prevention came into being when the drones were used by authorities to curb the spread of virus by spotting people with symptoms like high temperature in a crowd, predicting the future course of the disease and its likeability to recur. It was used for easy detection of red flags alarming the patients and healthcare authorities to make a faster decision. Maintainance of records, tracing and forecasting the nature of the virus from the available data on the media platforms like social media and predicting the number of positive cases and death analysis in any region so that proper curbing measures could be taken in time are other beneficial applications of AI. Anthropomorphic Robots and Service Robots were used to deliver essential services like cleaning and disinfecting of the hospital as well as the reception of the patients.

During the pandemic, it was also used for providing critical information on the networking sites for resource allocation and prioritising the decision making of the need of ventilators and respiratory supports for patients in the intensive care units and also to provide daily updates and trend analysis and charting of the course of treatment. ${ }^{8}$

COVID-19 detection neural network (COVnet) was used during a pandemic by the research scholars to differentiate between COVID-19 and community acquired pneumonia based on the visual 2D to 3D features interpreted from the volumetric chest CT scan. ${ }^{9}$ It was also used for developing vaccines in the clinical trials at an accelerated pace as normal testing and clinical trials required plenty of time and may not be possible humanly.

The use of smartphones and smart machines with variable step trackers, pulse oximeters and heartbeat monitors round the clock help in monitoring health through variables on personal devices. In the future field of Dermatology and Ophthalmology can use smartphones to collect images/photographs of eyes skin lesions wounds infections and help in medicating by addressing the shortage of specialists and reducing the time loss to diagnose those complaints. 
An AI system can assist physicians in providing up to date medical information from the latest journals, textbooks and clinical practices to provide proper patient care thus reducing the diagnostic and therapeutic errors that are inevitable in human clinical practice. ${ }^{10}$

\section{Limitations of Artificial Intelligence}

The lack of empathy due to machine to machine interactions or communications and automatic process of data collection makes customer disengagement and less intimacy. ${ }^{11}$

\section{CONCLUSIONS}

AI is an upcoming platform for identifying early infection and thus will help in fighting against other epidemics and pandemics in near future and can be used as a predictive and preventive health care strategy by developing useful algorithms. The biggest challenge to AI in the healthcare system is ensuring their adoption in daily clinical practice rather than whether the technology will be used occasionally. AI can have a direct and positive influence on the dynamics of the business world as patients sense of security will demand more of these smart machines in future for the betterment of people.

\section{REFERENCES}

1. Ericksson T, Biggi A, Bonera M. Think with me, or think for me? On the future role of artificial intelligence in marketing strategy formulation. Artificial intelligence in marketing strategy. 2020:795 -814

2. Davenport T, Kalakota R. The potential for artificial intelligence in healthcare. Future healthcare journal. $2019 ; 6(2): 94-98$

3. Vaishya R, Javaid M, Khan IH, Haleem A. Artificial intelligence application for COVID pandemic. Diabetics and metabolic syndrome: Clinical Research and Review. 2020;14:337 -339

4. McKinsey Global Institute. A future that works: automation, employment and productivity McKinsey global institute. 2017

5. Shahid MZ, Li G. Impact of artificial intelligence in marketing: a perspective of marketing professionals of Pakistan. $2019 ; 19$ (2): $1-8$

6. Russell SJ, Norvig P. Artificial intelligence: a modern approach. Pearson Education limited Malaysia. 2016

7. Bresnick J.Top 12 ways artificial intelligence will impact healthcare. HealthTAnalytics. 2018

8. Arora N, Banerjee AK, Narasu ML. The role of artificial intelligence in tackling COVID-19. Future virology. 2020:1-8

9. Li L, Qin L, Xu Z.et al. Artificial intelligence distinguishs COVID-19 from community acquired pneumonia on chest CT. Radiology. 2020: 1-16

10. Jiang F, Jiang Y, Zhi H, et al. Artificial intelligence in healthcare: past, present and future. Stroke and vascular neurology. $20172(4)$

11. Huang MH, Rust RT.A strategic framework for artificial intelligence in marketing. Journal of the Academy of marketing science. $2021 ; 49: 30-50$

12. Bryndin, Evgeniy. "Development of sensitivity and active behavior of cognitive robot by means artificial intelligence." International Journal of Robotics Research and Development 10.1: 1-11.

13. Senthiil, P. V., V. A. Sirusshti, and T. Sathish. "Artificial Intelligence Based Green Manufacturability quantification of a unit production process." International Journal of Mechanical and Production Engineering Research and Development 9.2 : 841- 
852.

14. Dange, Trupti, and Tarun Yengatiwar. "Drowsiness detection using artificial intelligence techniques." Science and Engineering (IJCSE) 2.4: 75-82.

15. Khan, Mudassir. "Big data analytics emerging trends, technology and innovations for the future business in the global market." International Journal of Scientific Research and Review 8.2: 745-750. 